\title{
Deep learning-based mixed-dimensional Gaussian mixture model for characterizing variability in
} cryo-EM

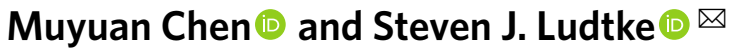

Structural flexibility and/or dynamic interactions with other molecules is a critical aspect of protein function. Cryogenic electron microscopy (cryo-EM) provides direct visualization of individual macromolecules sampling different conformational and compositional states. While numerous methods are available for computational classification of discrete states, characterization of continuous conformational changes or large numbers of discrete state without human supervision remains challenging. Here we present e2gmm, a machine learning algorithm to determine a conformational landscape for proteins or complexes using a three-dimensional Gaussian mixture model mapped onto two-dimensional particle images in known orientations. Using a deep neural network architecture, e2gmm can automatically resolve the structural heterogeneity within the protein complex and map particles onto a small latent space describing conformational and compositional changes. This system presents a more intuitive and flexible representation than other manifold methods currently in use. We demonstrate this method on both simulated data and three biological systems to explore compositional and conformational changes at a range of scales. The software is distributed as part of EMAN2.

C ryogenic-electron microscopy (cryo-EM) is used to image biological macromolecules in a near-native state and is capable of resolving structures to near-atomic resolution. However, most macromolecules possess substantial conformational and/or compositional variability as part of their biological function. In single-particle reconstruction (SPR), a micrograph contains a snapshot of many macromolecules, each frozen at a random point on its conformational and/or compositional landscape. This presents the difficulty that the features visible in any single structure solved using cryo-EM data will be limited by the conformational variability among the particles making it up. With more complete analysis, the presence of these variations can be turned into an advantage, as the individual data intrinsically explore a large portion of the conformational landscape of the system.

Many methods have been developed to address the heterogeneity problem in $\mathrm{SPR}^{1-3}$. Perhaps the oldest and most commonly used method is multi-model refinement/three-dimensional (3D) classification, in which multiple volumes are used as references and each particle is iteratively compared to the projections of each reference ${ }^{4-7}$. Focused classification is a variant of this method in which variability is explored only inside a user-defined mask ${ }^{8}$. These methods often work well when the system falls into a small number of discrete states, such as the two states associated with ligand binding. However, to work well, the number of discrete states should be small, and the quality of the initial seed volumes often has an impact on the results.

Another common practice is to perform multi-model refinement, then rely on a human to discard particles representing states judged to be 'bad',10. This process is typically iterated multiple times until a single map with improved resolution is achieved. This method produces one structure at high resolution representing the most populous state in the data, by simply ignoring contradictory data. This has the disadvantage of imposing human bias on the results, and while the resulting map has improved resolution, it clearly presents an incomplete picture of the macromolecule being studied.
In addition to 3D classification, multi-body refinement can be used to resolve local structural variability caused by conformational changes $^{11}$. This technique relies heavily on researchers' previous knowledge of structural domains in protein and requires the regions of interest to be large enough to provide sufficient signal for local orientation assignment.

Finally, we have seen the recent emergence of manifold embedding techniques to address the problem of structural variability ${ }^{12-16}$. These methods are fairly new and varied in their mathematical methods. While they have shown promising results, they also face difficulties in mapping the manifolds to biological interpretation, and the process of interpreting the structure at a point on the manifold is often time consuming.

In this paper, we present a strategy leveraging deep learning technology to map two-dimensional (2D) data directly to a $3 \mathrm{D}$ Gaussian mixture model (GMM). This produces a representation where conformational and compositional variations can be directly and intuitively related back to the data representation. A point on the manifold represents a specific Gaussian configuration that can be instantaneously visualized without needing to first reconstruct large subsets of particle data.

\section{Results}

The e2gmm method. One of the difficulties in SPR heterogeneity analysis is the mathematical representation of protein conformations. If we consider the motion of an object from position A to position B along a simple linear path, it should be possible to represent the position of the object on the path with a single value. However, when we represent this motion via images or volumes, the motion becomes a pattern of pixels becoming brighter and dimmer along the path in a complex sequence. Simple image analysis methods, such as principal component analysis (PCA), can readily identify the pixels involved in such a motion, but cannot readily map the highly nonlinear sequence of pixel variations back to the single degree of freedom we know exists in the underlying system. 


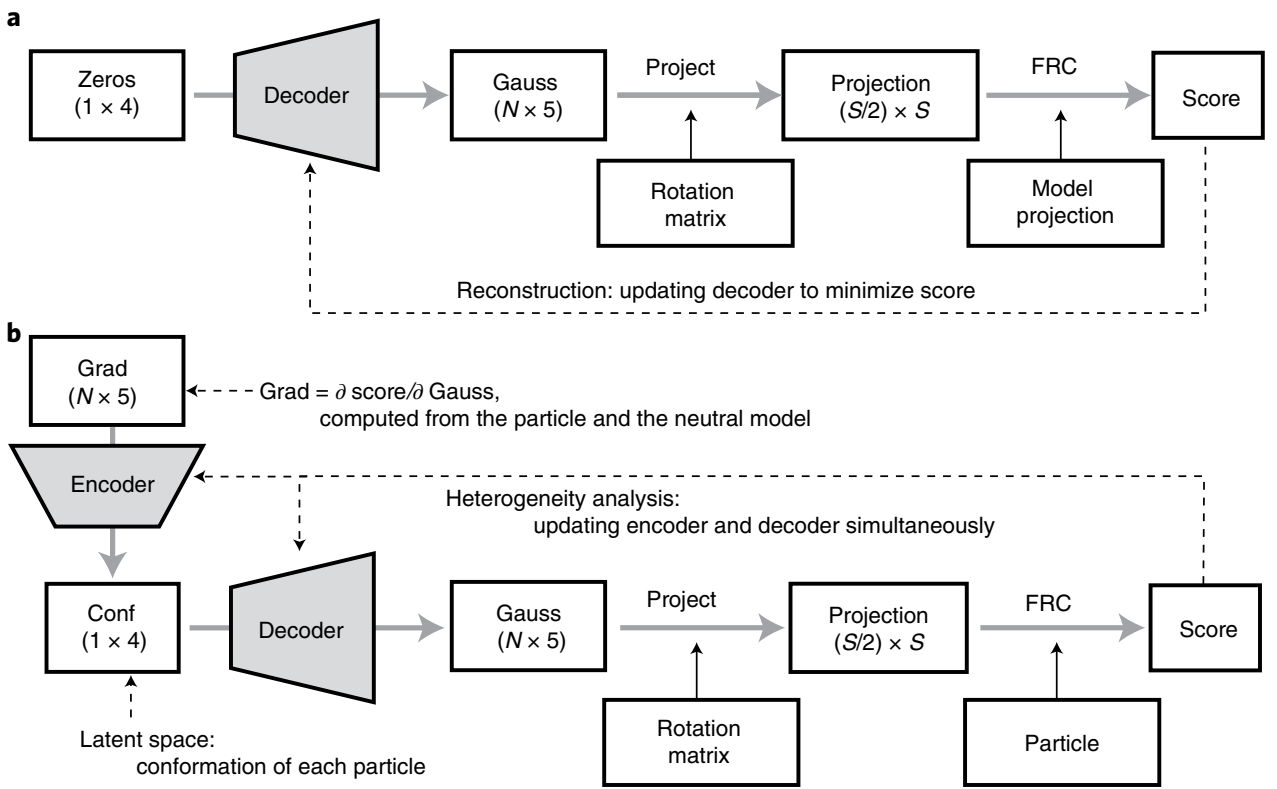

Fig. 1 | Neural network model. a, Training the decoder to represent the neutral map. b, Training the full network to represent system heterogeneity. Conf is the latent space vector representing the conformation of a particle. $S$ is the size of the particle in Fourier space.

Rather than attempting to determine paths in image space, we instead impose a Gaussian model at a specified level of detail, and then identify changes in Gaussian location and intensity that are self-consistent with the ensemble of particles. In this GMM, each function is defined by five variables: 3D coordinates, amplitude and width. The local motion of a domain is represented by a simple change in location of the Gaussians making it up, and the presence or absence of a ligand is represented by a change of amplitude.

Converting the Gaussian representation into an image representation (a projection) is a trivial process, whereas the inverse process, generating a GMM from a single projection image, is underdetermined. The inverse problem is sufficiently constrained only when a large ensemble of projection images in different orientations is considered. To solve this sparse and nonlinear inverse problem, we make use of deep learning methodologies. This design is completely unsupervised, and only requires the definition of a loss function describing the agreement between individual images and specific configuration of the GMM. We make use of the Fourier ring correlation (FRC) metric ${ }^{17}$ in the loss function, which has the additional advantage of being insensitive to microscope contrast transfer function (CTF) artifacts so long as the (phase flipped) images are reasonably stigmated with minimal drift.

The network design involves two components. First, a decoder, which maps a small latent vector, into a set of $5 \mathrm{~N}$ Gaussian parameters. The latent vector is simply a reduced dimensionality representation of the 3D configuration of the molecule. In linear analysis, each component in the latent vector represents one degree of freedom in the macromolecule. However, with the nonlinearity provided by the network, it is possible for local regions in the latent space to represent independent discrete states.

The second network component is the encoder, which maps 2D images, via their derivatives, into latent vectors. The latent vector then passes through the decoder to produce $5 \mathrm{~N}$ Gaussian parameters, which immediately provides a $2 \mathrm{D}$ projection or $3 \mathrm{D}$ volume as desired. This mapping process is constrained by the latent vector representation, and the set of particles mapped into this latent space will form a manifold, conceptually similar to other manifold methods in cryo-EM. However, due to the nonlinearities and our enforcement of a GMM with a specified level of detail, it also becomes possible to probe systems in very specific ways, which would be difficult using competing methods. For example, parameters of specific Gaussian components can be held constant, such that the GMM considers only variability in specific regions.

Our network structure is conceptually similar to an autoencoder ${ }^{18}$, in which the network is trained directly from raw data, with no need for ground truth. The goal of the autoencoder is to optimally match the input data to the same data at the output, after passing through a low dimensionality latent space. In our case, the input data are $2 \mathrm{D}$ particle data, and our network output is a complete 3D GMM. While this $3 \mathrm{D}$ model can recreate $2 \mathrm{D}$ projections for training, the GMM output is far richer than a $2 \mathrm{D}$ image. To achieve this result, a slightly different network training strategy is required.

We begin by training only the decoder so that it produces a single neutral 3D structure from a zero latent vector (Fig. 1a). The network is trained to produce $5 \mathrm{~N}$ Gaussian parameters best matching the provided neutral structure. The decoder is trained using an ADAM optimizer $^{19}$ with the FRC between the GMM and the provided map as the loss function (Methods). When trained, the decoder produces an accurate representation of the neutral map when given an input latent vector of zero.

Next, the encoder, which produces latent vectors from particle data, must be included in the training process (Fig. 1b). The goal of this procedure is for the latent space vector to represent as much of the variability of the specimen as possible. The training data consist of 2D particles and their orientation parameters from a standard single-particle refinement. The assigned orientations for the particles can be imported from a standard EMAN2 or Relion refinement $^{20,21}$. For each particle, we compute the gradient of the loss function between the particle image and GMM with respect to the neutral model GMM parameters. These gradients, $5 \mathrm{~N}$ parameters per particle, are the input to the encoder. The gradient vectors are computed in the coordinate system of the GMM, so they are invariant with respect to translation and rotation of the raw particles. The loss function is the FRC between the particle and Gaussian projection. For training, the encoder weights are initialized with small random values producing near zero latent vectors.

The particle data and Gaussian parameters will clearly not agree perfectly due to both noise present in the $2 \mathrm{D}$ particle 


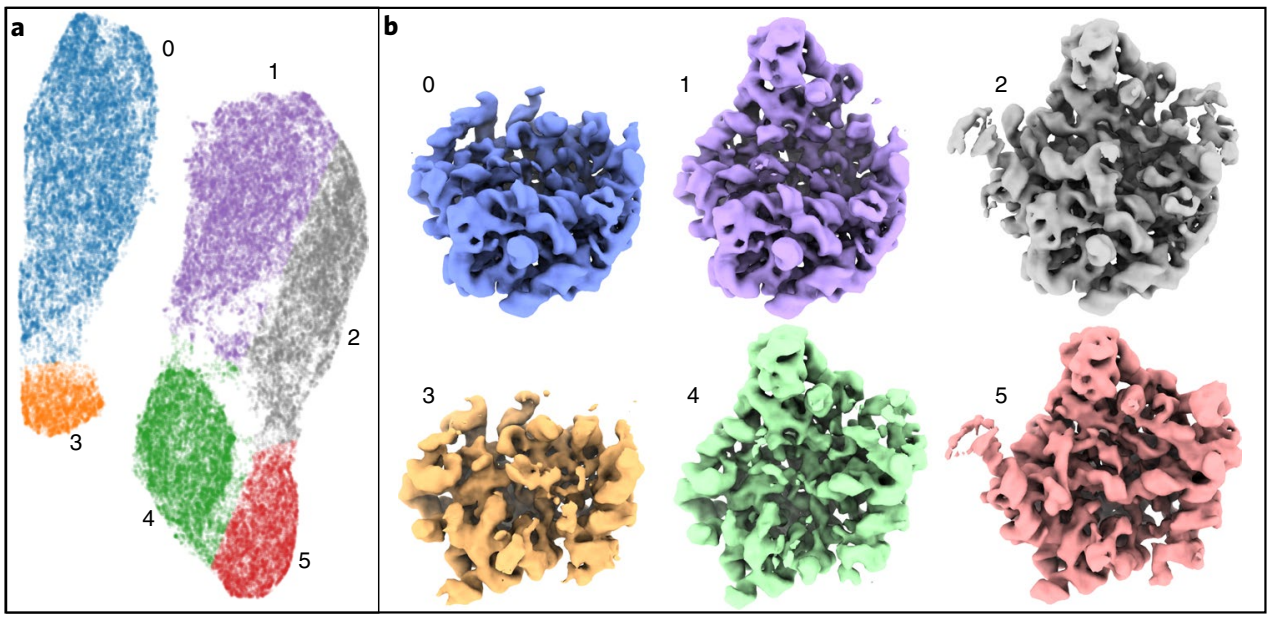

Fig. 2 Classification of assembling ribosomes. a, 2D embedding of particles from the 4D latent space, colored by labels from clustering. b, Averaged 3D structures produced using the 2D particles in each colored class, filtered to $8 \mathrm{~A}$ (Supplementary Video 1).

images as well as the conformational and compositional variability in the specimen. As noise is completely random within each particle, whereas the conformational and compositional variability follows patterns represented across many particles, the latent space should preferentially train for variations actually present in the data. We do not require the orientations to be truly optimal at this point, as when one part of the structure is moving with respect to another, the concept of a single correct orientation does not exist. Once the complete network has been trained to represent the variations in the data with the given orientations, another training cycle can be run where the particle orientations are refined against the dynamic GMM (Extended Data Fig. 1). This process can be iterated, although in practice it generally converges rapidly.

With a PCA representation of variability in image space ${ }^{22,23}$, the dimensionality of even a simple motion within a structure will be high since the motion involves many pixels undergoing nonlinear variations in intensity. With the GMM representation, each independent motion should require, at most, a single variable in the latent space. Thus, our default of a four dimensional (4D) latent vector can represent at least four independent variations. However, given the nonlinearity of the system and the fact that molecular variation tends to be highly constrained, it is readily possible for a single variable to possess multiple features across its domain. Additional dimensional reduction algorithms can be used on the latent space to further facilitate visualization. PCA applied to the latent space is one straightforward approach for visualization and segmentation. Even with the nonlinearity of the network, we still have the constraint that similar configurations will lie close to each other in the latent space and less similar configurations will be further apart. That is, we still expect continuous variations in structure to appear on manifolds in the latent space. Any latent vector can be visualized immediately via its GMM representation or by reconstruction of the particles in a local region in the latent space.

Application of e2gmm on cryo-EM datasets. We consider three publicly available Electron Microscopy Public Image Archive $(\text { EMPIAR })^{24}$ datasets, each of which exhibits different types of variability. Most observed variations are well known in each case, providing a validation of the method. We also observe additional motions not reported in the original studies, but generally consistent with our understanding of the underlying systems. As these are public datasets, experimental validation of these observations is clearly beyond the scope of this paper. Nonetheless, we believe these examples present the power of the method. Basic tests using simulated data are included in the Methods (Extended Data Fig. 5).

$50 \mathrm{~S}$ ribosome assembly. The bL17-depleted 50S ribosomal intermediate dataset (EMPIAR-10076) ${ }^{25}$ demonstrates the method's ability to identify discrete variability, such as partial complex formation/ligand binding. These data were also used in two other recent manifold method papers, permitting qualitative comparison of results ${ }^{14,15}$. We began with a structure determined using normal single-particle methods in EMAN2 to $3.3 \AA$ using the entire dataset excluding obvious ice contamination (124,900 particles). This structure was lowpass filtered to $8 \AA$, then used to generate a GMM with 3,082 Gaussians. The specific number of Gaussians was empirically determined, based on the targeted level of detail, and has little qualitative impact. Any Gaussians falling outside a specified mask can be excluded from the final model. Since most of the variations within this dataset are the presence/absence of individual ribosomal components, we initially permitted only the Gaussian amplitudes to vary. After training (Extended Data Fig. 3) we used UMAP (uniform manifold approximation and projection for dimension reduction $)^{26}$ to reduce the $4 \mathrm{D}$ space to $2 \mathrm{D}$ to visually explore the structural variability of the system. Particles were clearly separated into six visible clusters, each of which was reconstructed in $3 \mathrm{D}$. The observed structural differences recapitulate known states ${ }^{25}$ of ribosome assembly as shown in Fig. 2.

While the points form clear clusters in the $2 \mathrm{D}$ conformation space, such classification only represents large-scale structural differences and more subtle compositional changes can be observed within clusters. For example, we reconstructed the 2,000 particles closest to three linear points within one cluster, and the resulting structures show the introduction of h68-70 and h76-78 of the 23S ribosomal $\mathrm{RNA}^{25}$. Selecting three points along a similar line in a different cluster, one without the central protuberance domain, shows the introduction of the same rRNA helices (Fig. 3b).

Finally, we examined conformational changes within the system. One of the factors that limits the resolvability of the averaged ribosome is the smearing effect of the dynamic central protuberance domain. To study this, we continued training the network with the Gaussian positions also permitted to vary in this domain, including only particles where the central protuberance domain is present. This additional analysis identified a clear tilting motion of roughly $8^{\circ}$ of this domain (Fig. $3 \mathrm{~d}$ ). 

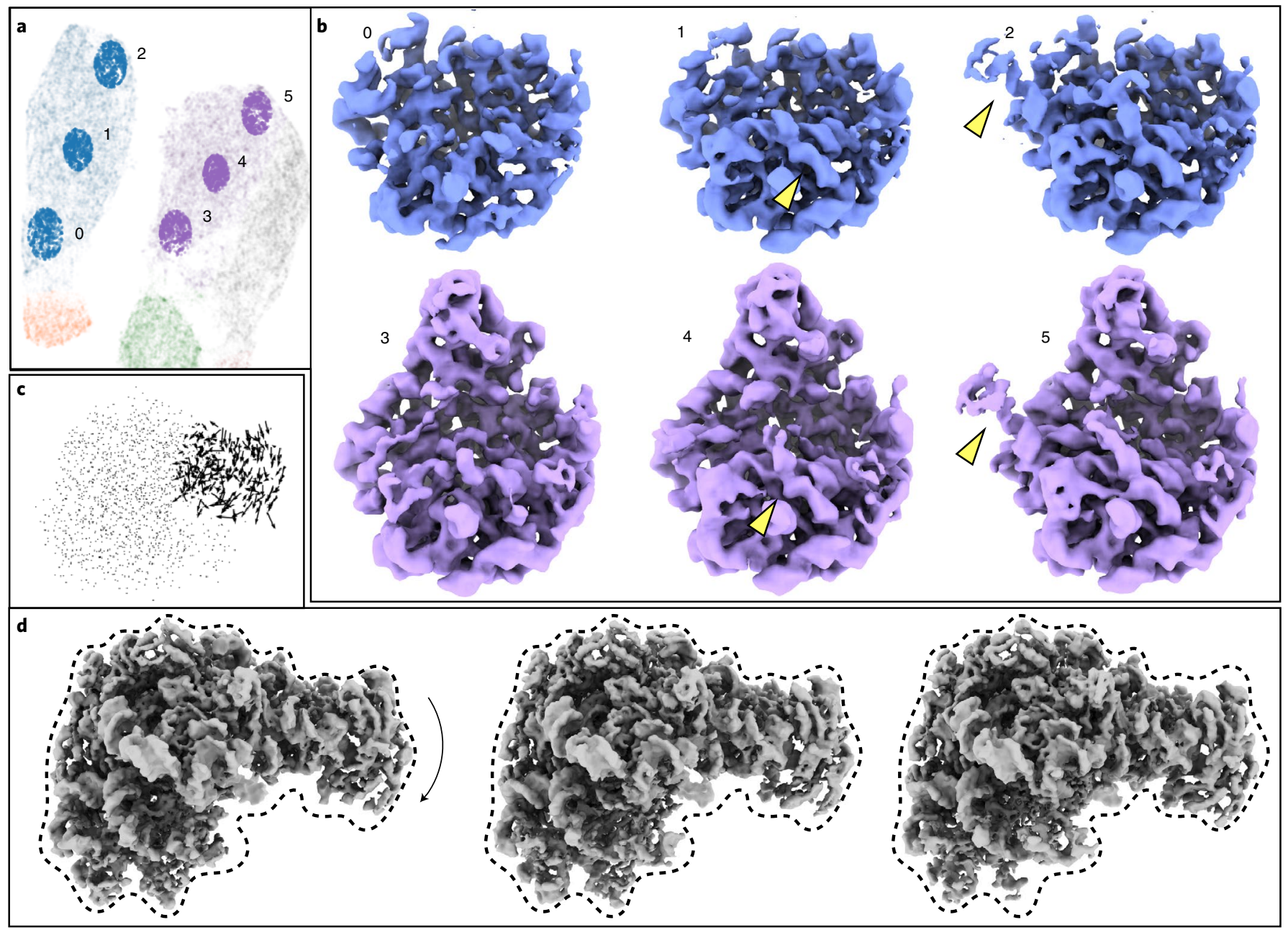

Fig. 3 | Exploration of subtle structural variability in the ribosome dataset. a, Location of particles sampled from the $2 \mathrm{D}$ embedding of the conformation space. b, Averaged structures reconstructed from the sampled particles. Yellow arrows point to the main differences between the structures. c, Motion trajectories of Gaussian coordinates in the central protuberance domain from the first eigenvector of conformational heterogeneity analysis. $\mathbf{d}$, Averaged structures of the particles at points along the motion trajectory. The dotted envelope is fixed to better visualize the changes in each map (Supplementary Video 2).

Spliceosome. The precatalytic spliceosome data (EMPIAR-10180) ${ }^{27}$ demonstrate large-scale conformational changes. We began with the particle orientation assignments and an averaged structure determined using EMAN2 to $4.6 \AA$ (327,490 particles). As resolution in cryo-EM is a measure of self-consistency rather than visible detail, it is possible to achieve relatively high measured resolutions in the presence of substantial motion blurring, even when the structure clearly lacks high-resolution detail. The density map was lowpass filtered to $13 \AA$ and represented by 2,048 Gaussians. All Gaussian parameters were allowed to vary. We used PCA to reduce the latent space to $2 \mathrm{D}$ for visualization of the subspace with the largest variation. Compared to nonlinear dimension reduction methods, PCA conveniently preserves the inverse transform so the eigenvectors can be mapped back to the Gaussian parameters and motion trajectories can be easily visualized. The first eigenvector from PCA shows a correlated motion of the helicase domain and the SF3b subunits, similar to the motion trajectories reported in previous studies.

While the eigenvectors from PCA exhibited several overall modes of motion of the complex, to better interpret the mechanism of the system it is more interesting to look at spatially localized eigenmotion trajectories. The use of PCA does not change the fact that the latent space has a nonlinear relationship to the motions of the system. Thanks to the characteristics of Gaussian models, we can focus on specific regions in real space. Rather than decomposing the point cloud in the latent space with PCA, we search for origin-crossing vectors in the latent space where the motion of Gaussian coordinates along the line is maximized in the domain of interest but minimized in rest parts of the protein (Fig. 4). Since the points from this dataset form a relatively isotropic distribution in the latent space, the movement represented by these vectors are nearly as important as the eigenmotion from PCA, while the Gaussian functions that are involved in the motion are far more localized. Furthermore, since the motion trajectories are localized in different domains, the two eigenmotion vectors are also orthogonal.

With the two independent eigenmotion vectors localized in the helicase and SF3b domains, we investigated the coordination of the two domains by looking at motion trajectories produced by the linear combinations of the two vectors. Adding the two vectors results in a motion mode that the two domains are moving toward the same direction, similar to the first eigenmotion extracted by PCA from the system. In the alternative combination, the two domains can be seen moving apart from each other, a motion mode never reported for the dataset (Fig. $4 \mathrm{f}$ and Extended Data Fig. 4). Note that the individual presented structures are the $3 \mathrm{D}$ reconstructions of particles near the corresponding point on the manifold. That is, unlike normal mode analysis, which predicts hypothetical 


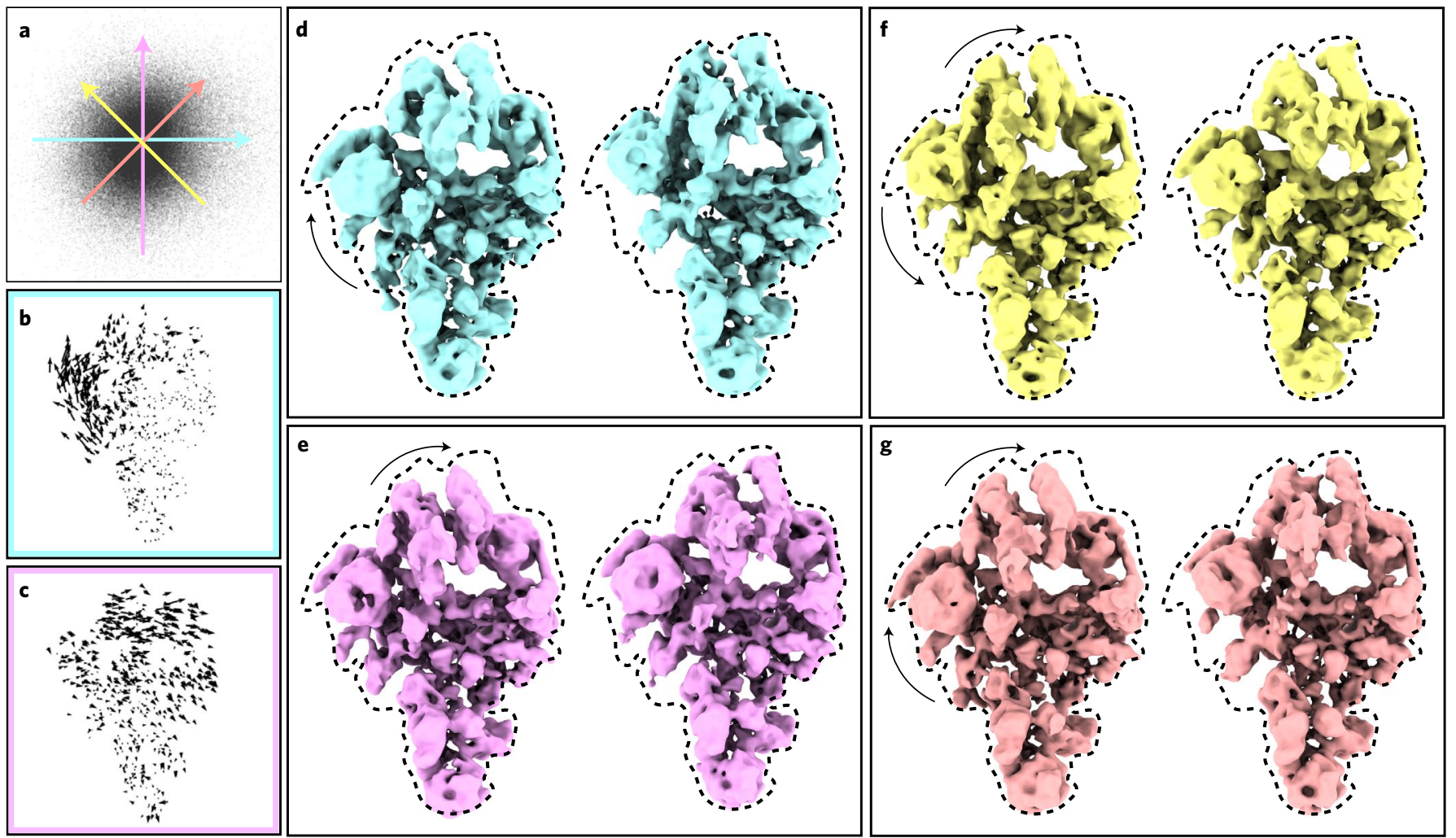

Fig. 4 | Structural variability analysis of spliceosome. a, Distribution of particles in the 2D space formed by the selected base vectors. Colored arrows correspond to different motion trajectories explored in panels with the corresponding color shown in $\mathbf{b}-\mathbf{g}$. Panels $\mathbf{b}, \mathbf{c}$ show motion vectors corresponding to the same-colored arrow in $\mathbf{a}$. The lengths of the vectors have been exaggerated for visualization. Panels $\mathbf{d}-\mathbf{g}$ show reconstructions of particles from along the corresponding colored vector in $\mathbf{a}$. The fixed dotted outline represents a fixed neutral reference to make visualizing the changes easier in static images. Arrows indicate the location and direction of strong visible motions in each example. Supplementary Video 3 presents the variabilites as motions in 3D for easier visualization.

modes with unknown amplitude and phase, in this case specific 3D structures are generated from the data for each putative point, demonstrating that each specific state can be generated from the data and that relative populations of different states can be considered, within the limits of noise.

SARS-CoV-2 spike protein. We use the spike structure of SARS-CoV-2 (EMPIAR-10492) for our third test. While the opening of the receptor binding domains (RBD) was not observed in the deposited particles due to the sucrose cushion used in sample preparation ${ }^{28}$, the RBDs in the published structure still have weaker density and lower resolution compared to the rest of the protein. In the original publication, $3 \mathrm{D}$ classification was performed, but only an asymmetrical structure with weak RBD was reported, and it was unclear what conformational changes caused the weakening of the RBD density.

To investigate this question, we performed heterogeneity analysis on the combined particle set of the RBD closed and weaker density state $(55,159$ particles). To demonstrate that e2gmm is directly compatible with other software, we directly used the averaged structure and particle orientations from the published Relion refinement and 2,188 Gaussian functions were used to model the averaged structure at roughly $7 \AA$. To break the C3 symmetry, we treated every particle as three copies in the symmetrical orientations. Only Gaussian functions in one asymmetric unit are allowed to vary, so every particle is mapped to three points in the conformation space, corresponding to the three asymmetric units.

After training, we performed PCA on the latent space and the eigenvectors show the motion of secondary structure elements at the RBD. Along the first eigenvector, the alpha helix at residue
335-344 can be seen tilting toward the RBD of its adjacent subunit by roughly $11^{\circ}$ (Fig. 5 and Extended Data Fig. 8). In the averaged structures along the same trajectory, the same helix in one of the neighboring subunits is undergoing the same motion, but in the opposite direction (Fig. $5 \mathrm{f}-\mathrm{h}$ ). Since the adjacent subunit was not targeted in the heterogeneity analysis, the presence of correlated motion suggests that the conformational changes of the RBDs in the two subunits are coordinated. Meanwhile, the same domain in the other subunit remains unchanged. On the other hand, the second eigenvector from PCA emphasizes the motion of the alpha helix at residue 364-371, as well as the beta-sheet strain at residue 354-359. Some coordination of motion in the adjacent subunit can also be observed but it is less clear.

In the density maps reconstructed from particles in specific conformations, the RBD at the subunit we focus on has stronger density than that of the other two subunits, suggesting the conformational changes we observe are indeed contributing to the weakening of density at the RBD (Extended Data Fig. 2).

\section{Discussion}

The main difference between the proposed method and most cryo-EM variability methods is the representation of the structure by a GMM, similar to methods sometimes used in coarsegrained modeling ${ }^{29,30}$. This is analogous to the idea of directly refining the atomistic structure against the raw data, but in a reduced representation based on the scale of the expected variations. This representation provides a number of advantages. First, it greatly reduces the number of parameters that needed to represent the molecule at any specified level of detail, limited by the sampling 


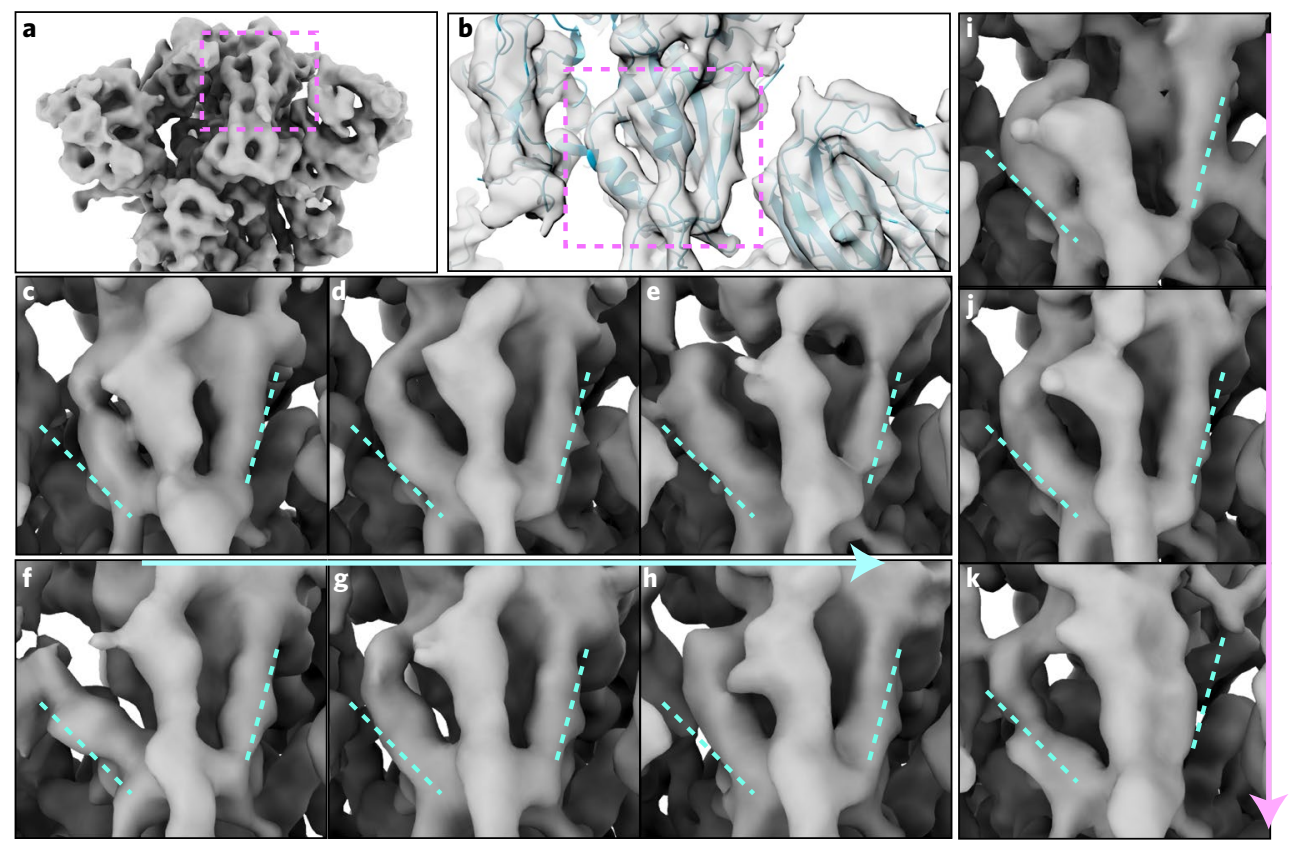

Fig. 5 | Structural variability analysis of the spike protein of SARS-CoV-2. a, Average structure of the spike protein, showing the RBD of the subunit that the analysis focuses on (dashed box). b, Density map of the target RBD overlaid with the molecular model (PDB 6zwv). c-e, Structures showing a sequence of targeted RBD structures along the first eigenvector. $\mathbf{f}-\mathbf{h}$, Structures showing a sequence of non-targeted RBD structures along the first eigenvector. i-k, Structures showing a sequence of targeted RBD structures along the second eigenvector. Supplementary Video 4 summarizes all of these changes dynamically for easier visualization.

of the image data ${ }^{31}$. For example, in the case of spliceosome, to represent the structure at $13 \AA$ using a voxel-based representation, the density map can be downsampled to a cube with a box size of 84 , so a total of 592,704 floating point parameters are required to represent the volume. Using the GMM, only 10,240 variables are needed for the 2,048 Gaussians used in the model, and the average Fourier shell correlation between the GMM and the density map is still above 0.95 for the spatial frequencies under consideration, indicating that it is a very good representation of the density map.

Second, at low resolution, Gaussian functions are a natural way to model cryo-EM maps ${ }^{32-34}$. If these methods were extended to atomic resolution representation, it may be necessary to include the atomic form factors and consider the differences between electronic potential and electron density, but at intermediate resolutions such subtleties are effectively undetectable. Typical protein structural variability, such as ligand binding and domain motion, can be easily represented as simple trajectories in the Gaussian parameter space. Whereas under voxel-based representations, a high-dimensional nonlinear model is required to depict the motion of a domain along even a linear trajectory, especially when the length of the path is longer than the size of the domain of interest. As a result, the complexity of the model required to describe the structural variability of the protein is greatly reduced, easing the effort to train the encoderdecoder neural networks.

Third, due to the mathematical characteristics of Gaussian functions in both real and Fourier space, our representation avoids the artifacts produced by common image processing operations. For example, to focus the analysis on a specific domain, only the parameters corresponding to Gaussians in that domain are allowed to change during network training. As all Gaussian functions still exist in the projection images, this will not introduce artifacts. The properties of Gaussian functions also ensure the model is always smooth at any snapshot during a continuous motion. Since the projection operation is performed by transforming the coordinates of the Gaussian functions, no interpolation artifacts are introduced by rotation or nonintegral translation. This also makes it easier to apply constraints in both spaces when studying the structural variability of proteins, such as focusing on specific domains in real space or limiting to a range of Fourier frequencies.

Finally, the use of Gaussian models makes the output from the neural networks directly and intuitively interpretable, unlike the typical abstract spaces produced by other manifold methods ${ }^{14,15}$. Each point in the conformational space is mapped to a set of Gaussian parameters, which corresponds to a complete 3D structure in one conformation. This means that for any given point, a representation can be generated either by reconstructing the particle image data in the vicinity of the point, or by directly converting the Gaussians into a density representation. The Gaussian map can provide a direct representation of the variations the network has learned, while the particle reconstructions can confirm that the actual 3D intermediate structures exist and agree with the Gaussians. For any two selected points in the confirmation space, it is easy to visualize the differences between those points by plotting a trajectory of coordinate motion or amplitude change. This can be especially useful in identifying putative changes when there are insufficient particles in the conformation of interest to provide a true $3 \mathrm{D}$ reconstruction at sufficient resolution. The Gaussian representation remains equivalently resolved at any point.

Unfortunately, some limitations remain in the current implementation of the method. First, since e2gmm requires the orientation of each particle as input, it only works in situations where a portion of the molecule is rigid enough that a reasonable neutral 3D structure exists, and reasonable particle orientations can be determined. While this is a safe assumption for most SPR cases, it is also possible that the protein complex of interest is so heterogeneous that the alignment in the initial refinement fails entirely, and particle-projection mismatch is caused by misalignment instead of conformational differences. A potential solution to this problem is simultaneous training of particle orientation and GMM conformation. 
While this is possible, training the model to convergence is more challenging when this approach is used.

Second, the protocol normally begins with an averaged structure of all particles, assuming this represents the 'neutral structure', which is then perturbed. This assumption is not always true. When a domain motion is large enough, regions in the averaged map may be sufficiently spread in space so that no Gaussian function is identified in that region when the neutral model is trained. As a result, the model excludes motion in that region since there are no Gaussians present to move. This can be corrected by selecting a better neutral structure with stronger density in this region. If dealing with a system with compositional variability, such as multiple ligands that may or may not be present, it is critical that the training volume be one with some density present for all ligands. This potential problem can also be reduced by building the neutral Gaussian model directly from aligned particles instead of the averaged structure, although this will incur a time penalty and may lead to a less robust model.

Finally, graphics processing unit (GPU) memory currently limits the size and resolution of the model. For example, a GPU with $11 \mathrm{~Gb}$ of memory supports up to 3,200 Gaussians with particles sampled at $128 \times 128$ pixels, and a batch size of eight during training. This would be sufficient to represent the $50 \mathrm{~S}$ ribosome at a roughly $8 \AA$ resolution, or smaller proteins at proportionally higher resolution. So, for many proteins, the method is currently limited to variations at the level of secondary structural features. This limitation is due to the Gaussian representation currently required by the underlying TensorFlow system. We expect that continuing evolution of GPU hardware as well as TensorFlow itself will remedy this problem in the near future.

Despite these minor limitations, e2gmm represents an easy to use mechanism for exploring macromolecular variability in cryo-EM with results that can be easily and intuitively interpreted. The user can define the resolution of interest, easily approaching features at any level of detail, within hardware limits. The next obvious development would be to operate on cryo-EM data, to permit similar studies in the context of the cellular environment, but technically this adaptation is not entirely straightforward to achieve due to the high noise levels in individual tilts and the increase in the amount of coordinated image data this would entail. All of the GMM operations are available through the program e2gmm_refine. py, and a graphical interface for interactive examination of results and exploring changes in parameters is provided by e2gmm.py. All of the necessary software is provided as part of EMAN2.91. A tutorial with sample data is available at http://eman2.org.

\section{Online content}

Any methods, additional references, Nature Research reporting summaries, source data, extended data, supplementary information, acknowledgements, peer review information; details of author contributions and competing interests; and statements of data and code availability are available at https://doi.org/10.1038/ s41592-021-01220-5.

Received: 25 January 2021; Accepted: 21 June 2021; Published online: 29 July 2021

\section{References}

1. Ludtke, S. J. Single-particle refinement and variability analysis in EMAN2.1. Methods Enzymol. 579, 159-189 (2016).

2. Scheres, S. H. W. Processing of structurally heterogeneous cryo-EM data in RELION. Methods Enzymol. 579, 125-157 (2016).

3. Jonić, S. Computational methods for analyzing conformational variability of macromolecular complexes from cryo-electron microscopy images. Curr. Opin. Struct. Biol. 43, 114-121 (2017).

4. Gabashvili, I. S., Agrawal, R. K., Grassucci, R. \& Frank, J. Structure and structural variations of the Escherichia coli $30 \mathrm{~S}$ ribosomal subunit as revealed by three-dimensional cryo-electron microscopy. J. Mol. Biol. 286, 1285-1291 (1999).
5. Scheres, S. H. W. et al. Maximum-likelihood multi-reference refinement for electron microscopy images. J. Mol. Biol. 348, 139-149 (2005).

6. Chen, D.-H., Song, J.-L., Chuang, D. T., Chiu, W. \& Ludtke, S. J. An expanded conformation of single-ring GroEL-GroES complex encapsulates an $86 \mathrm{kDa}$ substrate. Structure 14, 1711-1722 (2006).

7. Scheres, S. H. W. et al. Disentangling conformational states of macromolecules in 3D-EM through likelihood optimization. Nat. Methods 4, 27-29 (2007).

8. Penczek, P. A., Frank, J. \& Spahn, C. M. T. A method of focused classification, based on the bootstrap 3D variance analysis, and its application to EF-G-dependent translocation. J. Struct. Biol. 154, 184-194 (2006).

9. Lu, P. et al. Three-dimensional structure of human $\gamma$-secretase. Nature $\mathbf{5 1 2}$ 166-170 (2014)

10. Dong, Y. et al. Cryo-EM structures and dynamics of substrate-engaged human 26S proteasome. Nature 565, 49-55 (2019).

11. Nakane, T., Kimanius, D., Lindahl, E. \& Scheres, S. H. Characterisation of molecular motions in cryo-EM single-particle data by multi-body refinement in RELION. elife 7, e36861 (2018).

12. Fu, T. M. et al. Cryo-EM structure of caspase- 8 tandem DED filament reveals assembly and regulation mechanisms of the death-inducing signaling complex. Mol. Cell 64, 236-250 (2016).

13. Lederman, R. R. \& Singer, A. Continuously heterogeneous hyper-objects in cryo-EM and 3-D movies of many temporal dimensions. Preprint at https://arxiv.org/abs/1704.02899 (2017).

14. Zhong, E. D., Bepler, T., Berger, B. \& Davis, J. H. CryoDRGN: reconstruction of heterogeneous cryo-EM structures using neural networks. Nat. Methods 18, 176-185 (2021)

15. Punjani, A. \& Fleet, D. J. 3D variability analysis: resolving continuous flexibility and discrete heterogeneity from single particle cryo-EM. J. Struct. Biol. 213, 107702 (2021).

16. Dashti, A. et al. Retrieving functional pathways of biomolecules from single-particle snapshots. Nat. Commun. 11, 4734 (2020).

17. Van Heel, M. Similarity measures between images. Ultramicroscopy 21, 95-100 (1987)

18. Vincent, P., Larochelle, H., Bengio, Y. \& Manzagol, P.-A. Extracting and composing robust features with denoising autoencoders. In Proc. 25th International Conference on Machine Learning: ICML '08 1096-1103 (ACM, 2008); https://doi.org/10.1145/1390156.1390294

19. Kingma, D. P. \& Ba, J. ADAM: a method for stochastic optimization. Preprint at https://arxiv.org/abs/1412.6980 (2014).

20. Bell, J. M., Chen, M., Baldwin, P. R. \& Ludtke, S. J. High resolution single particle refinement in EMAN2.1. Methods 100, 25-34 (2016).

21. Zivanov, J. et al. New tools for automated high-resolution cryo-EM structure determination in RELION-3. eLife 7, e42166 (2018).

22. van Heel, M. \& Frank, J. Use of multivariate statistics in analyzing the images of biological macromolecules. Ultramicroscopy 6, 187-194 (1981).

23. Penczek, P. A., Kimmel, M. \& Spahn, C. M. T. Identifying conformational states of macromolecules by eigen-analysis of resampled cryo-EM images. Structure 19, 1582-1590 (2011).

24. Iudin, A., Korir, P. K., Salavert-Torres, J., Kleywegt, G. J. \& Patwardhan, A. EMPIAR: a public archive for raw electron microscopy image data. Nat. Methods 13, 387-388 (2016).

25. Davis, J. H. et al. Modular assembly of the bacterial large ribosomal subunit. Cell 167, 1610-1622.e15 (2016).

26. McInnes, L., Healy, J. \& Melville, J. UMAP: uniform manifold approximation and projection for dimension reduction. Preprint at https://arxiv.org/abs/ 1802.03426 (2018)

27. Plaschka, C., Lin, P.-C. \& Nagai, K. Structure of a pre-catalytic spliceosome. Nature 546, 617-621 (2017).

28. Ke, Z. et al. Structures and distributions of SARS-CoV-2 spike proteins on intact virions. Nature 588, 498-502 (2020).

29. Jonic, S. \& Sanchez Sorzano, C. O. Coarse-graining of volumes for modeling of structure and dynamics in electron microscopy: algorithm to automatically control accuracy of approximation. IEEE J. Sel. Top. Signal Process. 10, 161-173 (2016)

30. Birmanns, S. \& Wriggers, W. Multi-resolution anchor-point registration of biomolecular assemblies and their components. J. Struct. Biol. 157, 271-280 (2007)

31. Kawabata, T. Gaussian-input Gaussian mixture model for representing density maps and atomic models. J. Struct. Biol. 203, 1-16 (2018).

32. Kim, S. J. et al. Integrative structure and functional anatomy of a nuclear pore complex. Nature 555, 475-482 (2018).

33. Rout, M. P. \& Sali, A. Principles for integrative structural biology studies. Cell 177, 1384-1403 (2019).

34. Bonomi, M. et al. Bayesian weighing of electron cryo-microscopy data for integrative structural modeling. Structure 27, 175-188.e6 (2019).

Publisher's note Springer Nature remains neutral with regard to jurisdictional claims in published maps and institutional affiliations.

(c) The Author(s), under exclusive licence to Springer Nature America, Inc. 2021 


\section{Methods}

Gaussian representation of protein density maps. The GMM, $M$, is a simple sum of $N$ Gaussian functions in real space, $\bar{x} \in R^{3}$ :

$$
M(\bar{x})=\sum_{j=1}^{N} A_{j} \mathrm{e}^{-\frac{\left|\bar{x}-\bar{c}_{j}\right|^{2}}{2 \sigma_{j}^{2}}}
$$

Here, the Gaussian parameters are amplitude, $A_{j}$, width, $\sigma_{j}$, and center coordinates $\bar{c}_{j}$. In the network parameter space, the center parameters have a range $(-0.5,0.5)$, the amplitude has a range $(0,1)$ and the Gaussian width $(0.5,1.5)$. Note that only the relative values of the amplitude and width of the Gaussian functions within the model are meaningful, as the FRC metric is insensitive to overall brightness and filtration of the image. The center coordinates are scaled by the linear size of the image in pixels to form the projection images.

Internally, a projection orientation is a $3 \times 3$ rotation matrix irrespective of the stored orientation in terms of Euler angles, quaternions and so on ${ }^{35}$. In the equation that follows, we discard the $z$ component in the product, so $R$ is $2 \times 3$, excluding the $z$ row. A projection, $P$, of the GMM in $\bar{t} \in R^{2}$ is thus simply:

$$
P(\bar{t})=\sum_{j=1}^{N} A_{j} \mathrm{e}^{-\frac{\left|\bar{t}-\bar{R}_{c_{j}}\right|^{2}}{2 \sigma_{j}^{2}}}
$$

Our loss function is the FRC between the Fourier transform of $P$ and a particle image and $I$ in the same orientation. Note that the Fourier transform of $P$ can be computed by shifting the Gaussian sum to Fourier space for efficiency if the real-space representation is not required for some other purpose. The FRC between the Fourier transform of the GMM projection and a cryo-EM particle image is the average of the correlation coefficients over Fourier rings ${ }^{36}$ :

$$
\operatorname{FRC}(\mathcal{P}, \mathcal{I})=\frac{2}{b} \sum_{k=1}^{b / 2} \frac{\sum_{\theta} \mathcal{P}_{k, \theta} \cdot \mathcal{I}_{k, \theta}}{\sqrt{\sum_{\theta} \mathcal{P}_{k, \theta}^{2} \cdot \sum_{\theta} \mathcal{I}_{k, \theta}^{2}}}
$$

where $b$ is the box size in pixels, $k, \theta$ are fast Fourier transform (FFT) polar coordinates and $\mathcal{P}$ and $\mathcal{I}$ are the FFTs of $P$ and $I$. As this is an operation over the FFT of discrete images, the sum over $\theta$ covers all values at $k \pm 0.5$ pixels. Since each ring is an independently normalized dot product, the FRC is insensitive to multiplication by any nonzero radial (filter) function. So long as the CTF phases have been correctly flipped and astigmatism and drift are minimal, CTF amplitude correction can be ignored. While the signal to noise ratio will be lower in the particle at points where the CTF amplitude is low, the FRC will still be maximized for an individual particle when the GMM best agrees with the underlying particle density irrespective of CTF

Neural network structure and parameter selection. The structure of neural networks and the parameters during the two phases of training process are user adjustable, but the defaults are suitable for most use cases. By default, the encoder and decoder both have three fully connected hidden layers, each with 512 units. A dropout layer with a rate of 0.3 , as well as a batch normalization layer is included before the final output layer of both networks (Extended Data Fig. 7). The ReLu function is used for activation in each layer, except for the output layer of the decoder that uses a sigmoid activation function. During the training process, the default learning rate is 0.0001 , with an $\mathrm{L} 2$ regularization of 0.001 . A small random variable is also added to the latent space vector before it is input to the decoder as a way to enforce the continuity of particle distribution in the latent space. This is similar to the concept of variational autoencoder, except that the variation of the random variable is not trainable here. An additional regularization factor is applied to the standard deviation of the amplitude and width of Gaussian functions to encourage the Gaussian functions to spread out in real space.

The number of Gaussian functions used in the model is decided based on the size of molecule and the target resolution. In practice, to build a Gaussian model from a density map, we start from a small amount of Gaussian functions (for example, 256) and target a low resolution (for example, $50 \AA$ ), and run the decoder optimization until the FRC curves between projections of the Gaussian model. The projections of the density map below the target resolution are always above 0.95 . Then, we double the number of Gaussian functions, increase the target resolution and repeat the process. When increasing the number of Gaussian functions, each newly added Gaussian will be seeded near an existing one, so the low-resolution correlation between the Gaussian model and the density map from the previous round is roughly preserved. Typically, within 3-5 rounds, the decoder can produce a Gaussian model that matches the density map at the target resolution. When a user-defined mask is provided, the program will exclude Gaussian functions whose centers fall out of the mask, resulting in slightly fewer Gaussian functions than the targeted number.

Using the trained decoder, is possible to visualize any point in the latent space, or a derived reduced representation if it can be mapped back to the latent space. It is sometimes also useful to display the vector motions connecting two points in the latent space for all Gaussians. This can be easily presented as a quiver plot, with a vector drawn for each Gaussian between its position at point $\mathrm{A}$ in the latent space to its position at point $\mathrm{B}$ in the latent space. If the motions are particularly small compared to the size of the molecule, an optional scaling factor can be used to make the vectors more visible. A graphical tool, e2gmm_analysis.py, is provided to easily generate such plots.

Tests on simulated datasets. To verify the method's fundamental capabilities, testing was performed on three simple simulated datasets (Extended Data Fig. 5), each consisting of random projections of a dynamic 3D model with a small amount of added noise. The first system included a large rigid domain with a smaller domain undergoing linear motion. The path length of the linear motion was longer than the width of the moving domain. Twenty $3 \mathrm{D}$ density maps were generated along the trajectory and 200 particles were generated for each $3 \mathrm{D}$ map, by projecting the map in a random orientation and adding a small amount of noise. In the simulation, we simply used the known projection orientations since the routine is normally used with predetermined orientations. For simplicity, in this example we use a one-dimensional latent space to avoid the need for any further dimensional reduction. After training the GMM, the resulting latent variable has good agreement with the location on the path. A plot of true conformation versus the single latent variable is shown in Extended Data Fig. 5d.

It is worth noting that, even for this simple system, the estimated particle conformation distribution includes some off-diagonal points that will tend to be biased toward zero, the neutral conformation. This is because the simulated domain movement occurs in a plane, so in some orientations the motion is effectively unobservable. In such cases, there is a bias toward the neutral state. While this artifact is unavoidable and populating the manifold with particles will produce some near the origin of the latent space irrespective of their true conformation, this does not mean the manifold itself is inaccurate. So long as orientations are sufficiently diverse, the manifold should still be accurately determined. Indeed, with some effort it may be possible to remove such outliers from the particle distribution by testing whether the change in GMM would be detectable in the orientation of each individual particle.

In the second example, we simulated the cyclic rotation of a small domain around an axis, to show the method can learn nonlinear/cyclic motion trajectories. In the simulated dataset, 36 density maps were generated along the movement trajectory and 200 particles were used for each snapshot. Here, we used a 2D latent space, so the motion could be directly modeled by the encoder with no further dimensional reduction. After training, the particles distribution in the latent space roughly formed a circle (Extended Data Fig. 5f), and when viewed in polar coordinates, the angle of each point in the latent space correlates well with its ground truth rotation angle of the small domain (Extended Data Fig. 5g).

Finally, we demonstrate the performance of the method when the system contains a mixture of conformational and compositional heterogeneity. The domain motion in this simulated system is the same as the first example, but for half of the population, we added a small additional density to the map, to represent compositional variability (Extended Data Fig. 5h). The compositional difference and the domain motion are independent. A Gaussian model was built from the averaged density map and trained to embed the particles onto a $2 \mathrm{D}$ latent space. After training, particles form two curves on the latent space that are roughly parallel to each other. Comparing to the ground truth conformation of the particles, it is clear that points on the two curves represent particles with and without the extra density, and the trajectory along the curve represents the linear motion of the flexible domain (Extended Data Fig. 5i). This also highlights the ability to separate compositional and conformational heterogeneity within the system.

\section{Additional data processing details for tests on real data. For the ribosome} dataset, obvious ice contamination was removed using the EMAN2 neural network particle picking tool before refinement. Single model refinement was performed using the remaining particles, which were split into two independent subsets. As we were not attempting to test the refinement pipeline, a high-resolution structure (EMD-8455) was lowpass filtered and phase randomized beyond $20 \AA$ to serve as an initial model for the refinement.

For the spliceosome dataset, all provided particles were used in the single model refinement. A high-resolution structure (EMD-3683) was lowpass filtered and phase randomized beyond $25 \AA$ to serve as the initial model for the refinement.

For the SARS-CoV-2 spike protein dataset, Phenix real-space refinement was performed to produce atomic models of the RBD for each frame of the continuous motion. Each density map was lowpass filtered to $5 \AA$, and the RBD of the target asymmetrical unit was segmented in UCSF Chimera using the Protein Data Bank (PDB) model 6ZWV. Real-space refinement was performed using the segmented RBD domains and the PDB model as the starting point.

Reproducibility. Since the method includes stochastic components, it is worth considering reproducibility. Toward this end, we tested the analysis of the EMPIAR-10076 dataset by evenly separating the data into even/odd subsets. The entire processing pipeline, including single model refinement, the generation of 
Gaussian model and the heterogeneity analysis, was performed independently on each subset. The GMM parameters and the training process for the two subsets were the same as described for the full dataset.

While the learned spaces are not identical due to the stochastic nature of the process, the number of clusters, the arrangement of the clusters on the manifold and the number of particles within each cluster are equivalent (Extended Data Fig. 6). Further, after clustering the particles and reconstructing a 3D structure for each class, we can find a one-to-one match between the 3D class averages from the two subsets. The structures of the matched classes from particles different subsets are highly consistent, and Fourier shell correlation between the corresponding structures extend beyond $4 \AA$.

This test establishes that functional reproducibility, while not guaranteed, is clearly possible in this method. We suggest that this even/odd split test, similar to the 'gold standard' methods used for resolution testing in single model refinement, represents a reasonable test of the reproducibility of biological conclusions drawn from the results of the method.

Computational requirements. For EMPIAR-10076, starting from a completed single-particle refinement, the first round of heterogeneity analysis, which focuses on only the amplitude changes of Gaussians required around $3 \mathrm{~h}$ on a GeForce RTX 2080 TI GPU, including the training of the GMM model and the dimension reduction process. Less than $1 \mathrm{~h}$ on a 12 -core workstation was required to embed the encoder latent space in $2 \mathrm{D}$, perform clustering, and reconstruct all of the $3 \mathrm{D}$ density maps. The second round of heterogeneity analysis focusing on the conformational change in the central protuberance domain also required roughly $3 \mathrm{~h}$ on the GPU and $<1 \mathrm{~h}$ on the 12 -core workstation.

For the EMPIAR-10180 dataset, the heterogeneity analysis required roughly $3 \mathrm{GPU}-\mathrm{h}$ and $30 \mathrm{CPU}-\mathrm{h}$ for the reconstruction of the density maps along the four reported motion trajectories.

The provided Relion alignment was used for the EMPIAR-10492 dataset. Heterogeneity analysis required roughly 2 plus $10 \mathrm{CPU}-\mathrm{h}$.

Comparison to existing methods. The recently published heterogeneity methods, CryoDRGN $^{14}$, CryoSPARC 3DVA ${ }^{15}$ and e2gmm (this paper), all made use of the ribosome (EMPIAR-10076) and spliceosome (EMPIAR-10180) as two of their examples, permitting users interested in comparing the methods to draw their own conclusions. While the results produced by the different packages on the two datasets are similar in general, the three methods use very different approaches to solve the same problem and each has its own advantages, which we discuss briefly.

3DVA solves the structural variability of the protein complex using a linear subspace model. The nature of the method makes it difficult to represent large-scale motions, where the trajectory of the conformational change is not linear with respect to the intensity of individual voxels. On the other hand, the linearity constraint also greatly simplifies the problem. So, when the heterogeneity within the system meets the linearity criteria, often the case in single-particle analysis, it can produce accurate results very quickly. For example, to solve the motion within the spliceosome dataset, 3DVA takes roughly $3 \mathrm{GPUh}$, similar to e2gmm processing time, but is $20 \times$ faster than CryoDRGN. Its performance is best demonstrated in the ribosome assembly dataset, as the compositional variability within the complex is strictly linear with the intensity of the voxels. Compared to our approach (Extended Data Fig. 3), the separation of classes is more obvious in their linear subspace, even without the extra UMAP embedding step. GMM clustering on the 3DVA latent space shows seven ribosome classes, and six of them directly match the six classes from our result. The extra class identified by $3 \mathrm{DVA}$ is similar to the subtle changes from our result shown in Fig. 2b, which do not form obvious clusters in the conformation space but can still be resolved in e2gmm with further analysis.

CryoDRGN uses an encoder-decoder deep neural network architecture conceptually similar to ours, but the underlying data representation uses a classical coordinate-based approach on the 3D density map. Compared to e2gmm, one advantage of CryoDRGN is its capability to generate neutral state structures from particles with predetermined orientations, without the need for a reference $3 \mathrm{D}$ density map. This makes it possible to obtain distant states that are not covered in the averaged structure from the single-particle refinement. For example, in the $50 \mathrm{~S}$ ribosome assembly example, CryoDRGN is able to capture the small cluster of $70 S$ ribosome ( $<1 \%$ of particles), an impurity of the dataset, in the embedded conformational space that was not immediately detected in the results from 3DVA or our software.

In e2gmm, our use of a GMM representation has numerous advantages, including a reduction in time and resource requirements. In the same benchmark datasets, e2gmm is roughly as fast as 3DVA and is $10-20 \times$ faster than CryoDRGN, while producing qualitatively similar results. Also note that the tests of our software are performed on a consumer grade GPU (GTX2080), which only has roughly a third of the memory and substantially lower performance than the hardware used in the CryoDRGN and 3DVA benchmarks.
One of the main difficulties in analyzing protein heterogeneity using other 'manifold methods' is to interpret the particle distribution on the manifold and draw biological conclusions from the results. Generally, to interpret the structural difference between any two points on a manifold requires identifying particles near both points and reconstructing them in 3D. With e2gmm, we can put any latent vector into the decoder and immediately have a set of Gaussian coordinates to display on the screen. The user can literally drag the mouse around the latent space and observe the changes in the underlying Gaussian model interactively. Further, with e2gmm a mask can be used to define a subset of Gaussians to model during network training. Since the underlying Gaussian model is completely smooth, doing this does not introduce any edge artifacts into the system. While the capability of representing particles on the determined manifold is similar across all of these methods, with e2gmm it is much easier to find specific paths in a potentially multidimensional manifold that correspond to specific variations of interest. Thanks to the advantages, we are able to identify conformational changes from the two datasets that are not described in the previous work, such as the tilting of the central protrusion domain of the ribosome, and the independent movement of the helicase and SF3b domains in the spliceosome dataset.

Reporting Summary. Further information on research design is available in the Nature Research Reporting Summary linked to this article.

\section{Data availability}

The three datasets used in the paper are publicly available through EMPIAR. The 50S ribosomal intermediate dataset is acquired from EMPIAR-10076, the spliceosome dataset is acquired from EMPIAR-10180 and the SARS-CoV-2 spike protein dataset comes from EMPIAR-10492. Structures produced in this paper are deposited in Electron Microscopy Databank (EMD): EMD-24129, classification of 50 S ribosome assembly states (Fig. 2); EMD-24130, subtle structural variability of ribosome assembly intermediates (Fig. 3b); EMD-24131, continuous movement of the central protuberance domain of 50S ribosome (Fig. 3d); EMD-24092, EMD-24093, EMD-24094 and EMD-24096, the four movement modes of spliceosome (Fig. 4); and EMD-24118 and EMD-24119, the two movement modes of SARS-CoV-2 spike protein RBD (Fig. 5). Note that the main data file for each entry contains only one representative class or video frame; the entire $3 \mathrm{D}$ video or classification result is deposited as multiple 3D maps in the additional data files of each EMD entry.

\section{Code availability}

EMAN2.91 is free and open source software available from http://eman2.org with source code on GitHub (https://github.com/cryoem/eman2).

\section{References}

35. Baldwin, P. R. \& Penczek, P. A. The transform class in SPARX and EMAN2. J. Struct. Biol. 157, 250-261 (2007).

36. Harauz, G. \& van Heel, M. Exact filters for general geometry three dimensional reconstruction. Optik 78, 146-156 (1986).

\section{Acknowledgements}

This work was supported by National Institutes of Health grant no. R01GM080139 to S.J.L. and computational resources were provided by BCM's CIBR center for Computational and Integrative Biomedical Research.

\section{Author contributions}

M.C. and S.J.L. designed the method and wrote the paper.

\section{Competing interests}

The authors declare no competing interests.

\section{Additional information}

Extended data are available for this paper at https://doi.org/10.1038/ s41592-021-01220-5.

Supplementary information The online version contains supplementary material available at https://doi.org/10.1038/s41592-021-01220-5.

Correspondence and requests for materials should be addressed to S.J.L.

Peer review information Nature Methods thanks Timothy Grant and the other, anonymous, reviewer(s) for their contribution to the peer review of this work. Arunima Singh was the primary editor on this article and managed its editorial process and peer review in collaboration with the rest of the editorial team.

Reprints and permissions information is available at www.nature.com/reprints. 


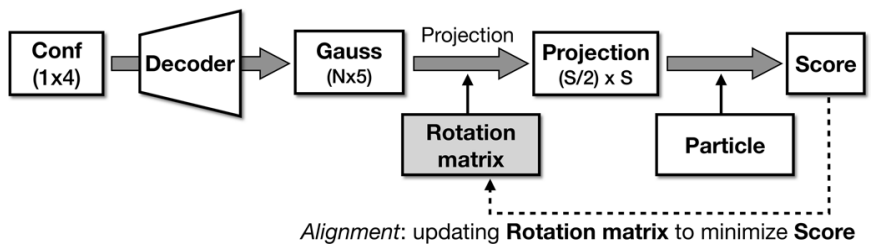

Extended Data Fig. 1 | Workflow for local particle orientation refinement. Workflow for local particle orientation refinement using the trained Gaussian model. This process can optionally be used after training the full GMM to improve particle orientations. 

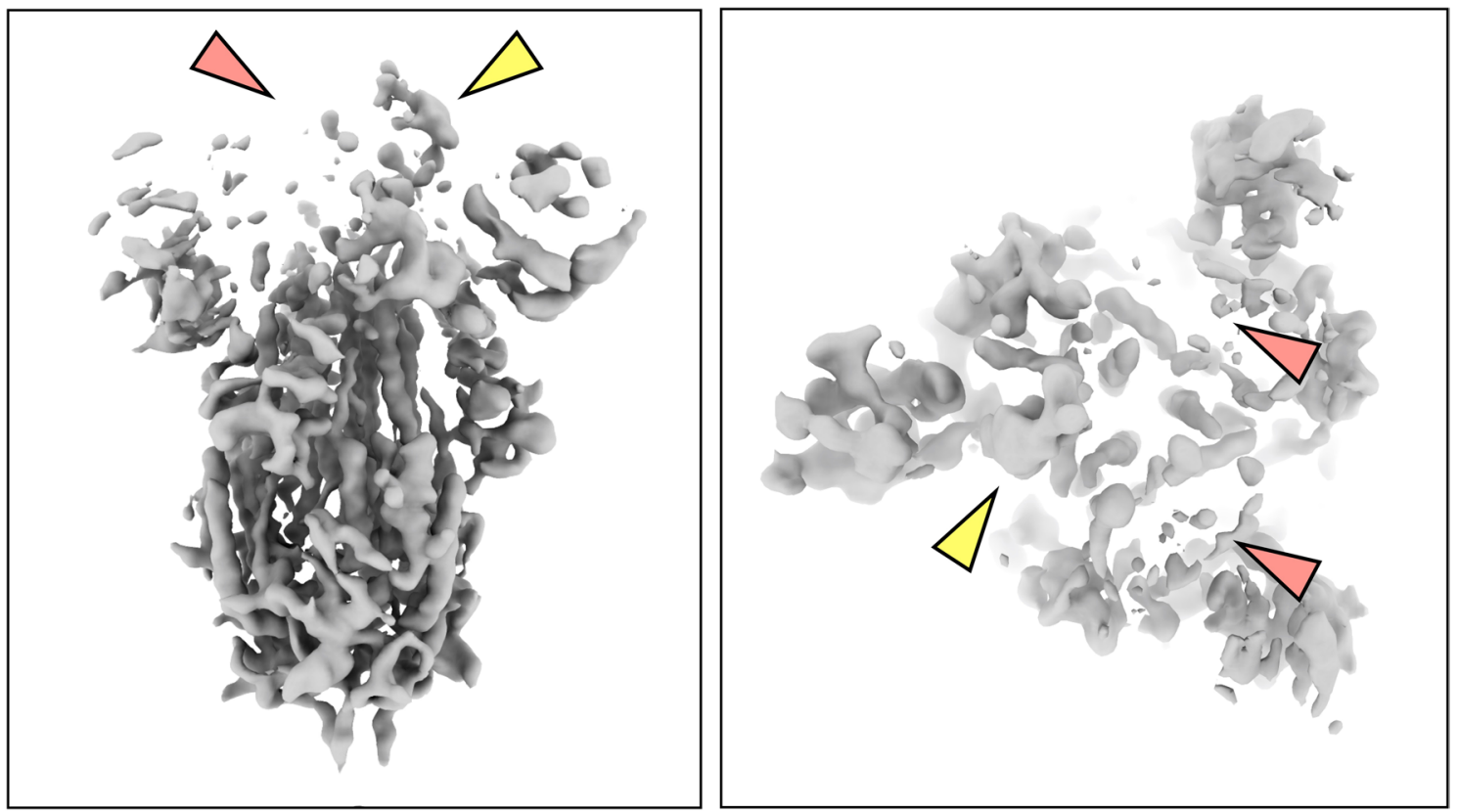

Extended Data Fig. 2 | Structure of SARS-COV2 spike protein visualized at high isosurface threshold. Structure of SARS-COV2 spike at one point in the continuous motion, visualized at high isosurface threshold. Note that the RBD of the subunit the heterogeneity analysis focuses on (yellow arrow) is still solid while the other two RBDs (red arrows) already vanish. This suggests the continuous motion is contributing to the weakening of density at the RBD. 


\section{NATURE METHODS}

ARTICLES
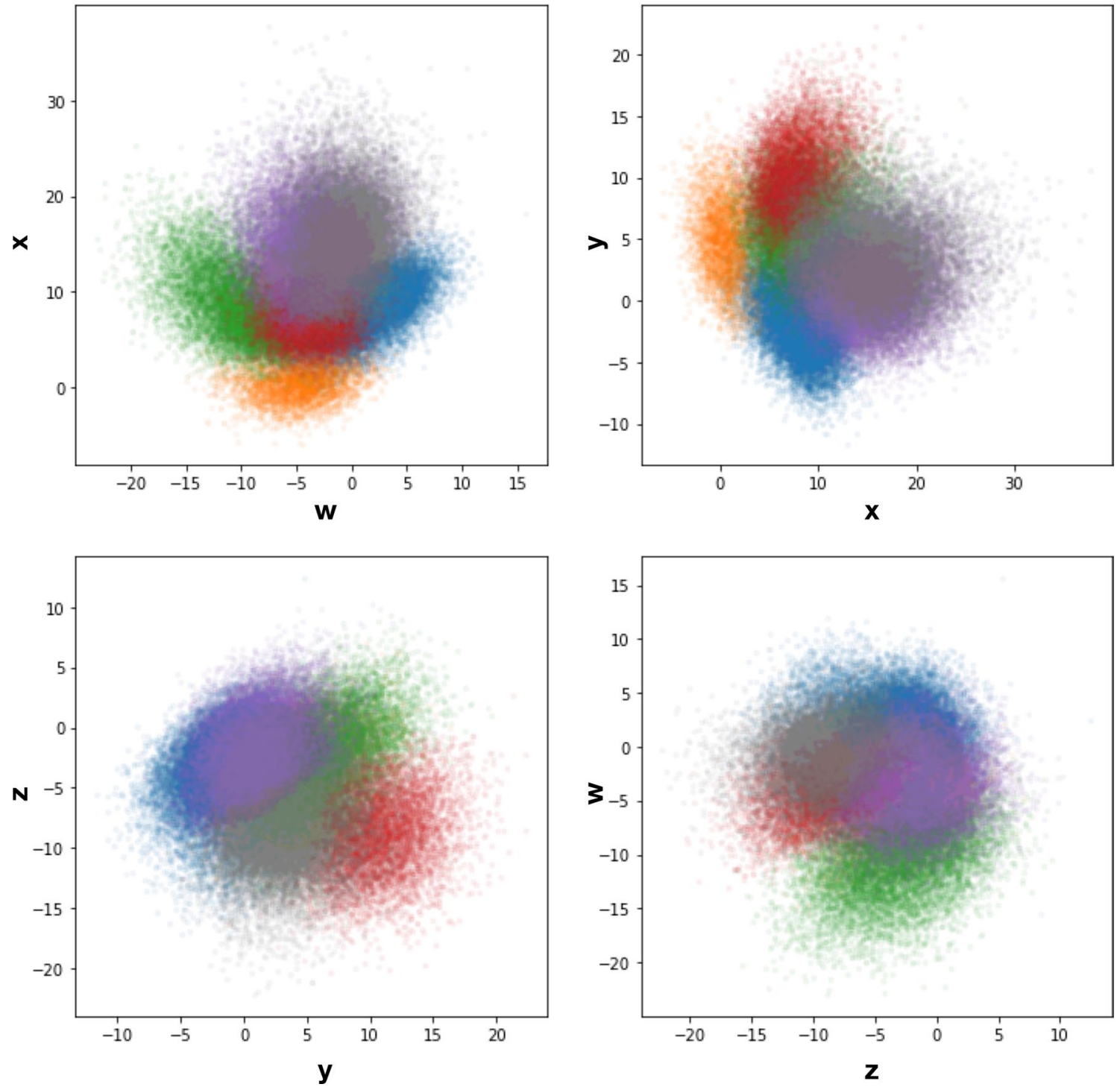

Extended Data Fig. 3 | 50 S ribosome particle distribution in the 4D encoder latent space. 50S ribosome particle distribution in the 4D encoder latent space, colored by the classification results shown in Fig. 2. 
ARTICLES

NATURE METHODS
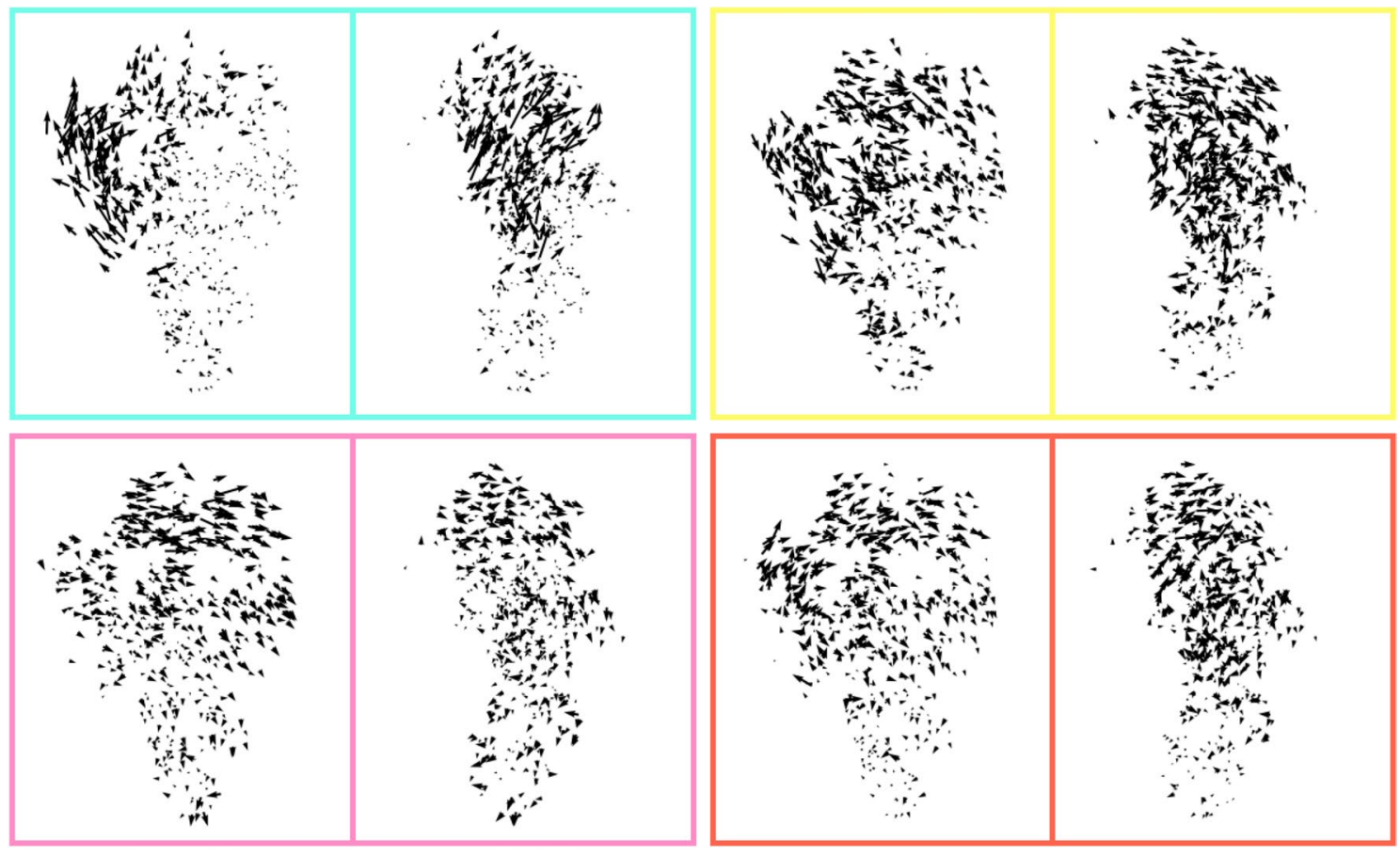

Extended Data Fig. 4 | Motion trajectory vectors of the spliceosome. Front and side views of the motion trajectory vectors from the four identified motion modes of the spliceosome dataset shown in in Fig. 4 deg.

NATURE METHODS | www.nature.com/naturemethods 

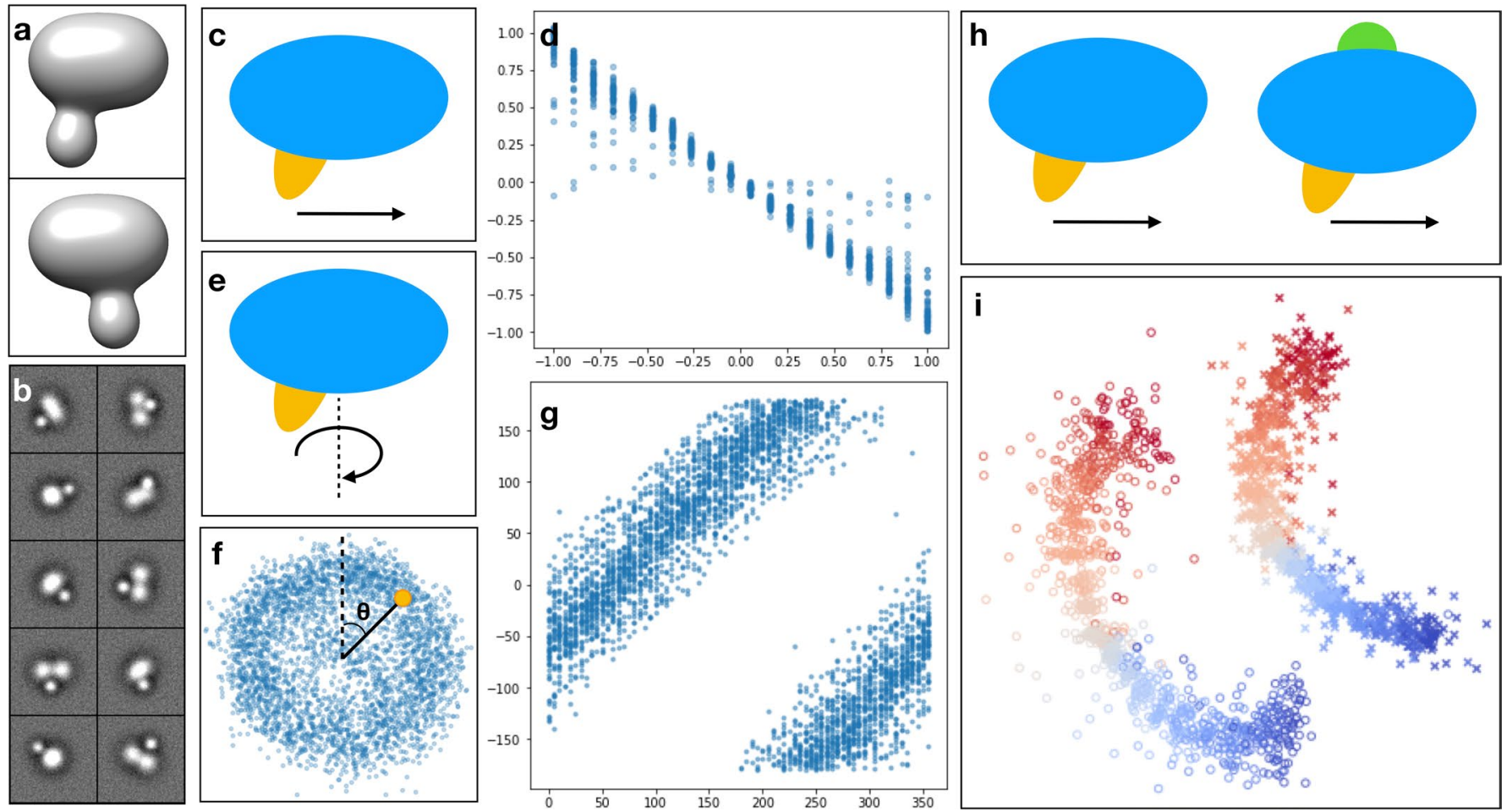

Extended Data Fig. $\mathbf{5}$ | Results on simulated datasets. Results on simulated datasets. a, 3D view of two snapshots of the simulated system at different frames of the movement trajectory. b, Sample simulated particles. c, Model of linear domain motion (yellow). d, Scatter plot of the ground truth position vs estimated particle conformation of the linear domain movement. $\mathbf{e}$, Model of domain rotation around an axis. $\mathbf{f}$, Estimated particle distribution of (e) on the $2 \mathrm{D}$ latent space. $\mathbf{g}$, Scatter plot of the ground truth rotation angle vs estimated particle angle in the latent space $(\theta$ in $\mathbf{f})$. $\mathbf{h}$, Combination of independent linear domain motion (yellow) and compositional change (green). $\mathbf{i}$, Particle distribution of (h) on the 2D latent space. Points are colored by their ground truth position along the linear domain motion trajectory. Particles with the extra density are marked as ' $x$ ', and the rest are marked as ' $\mathrm{o}$ '. 

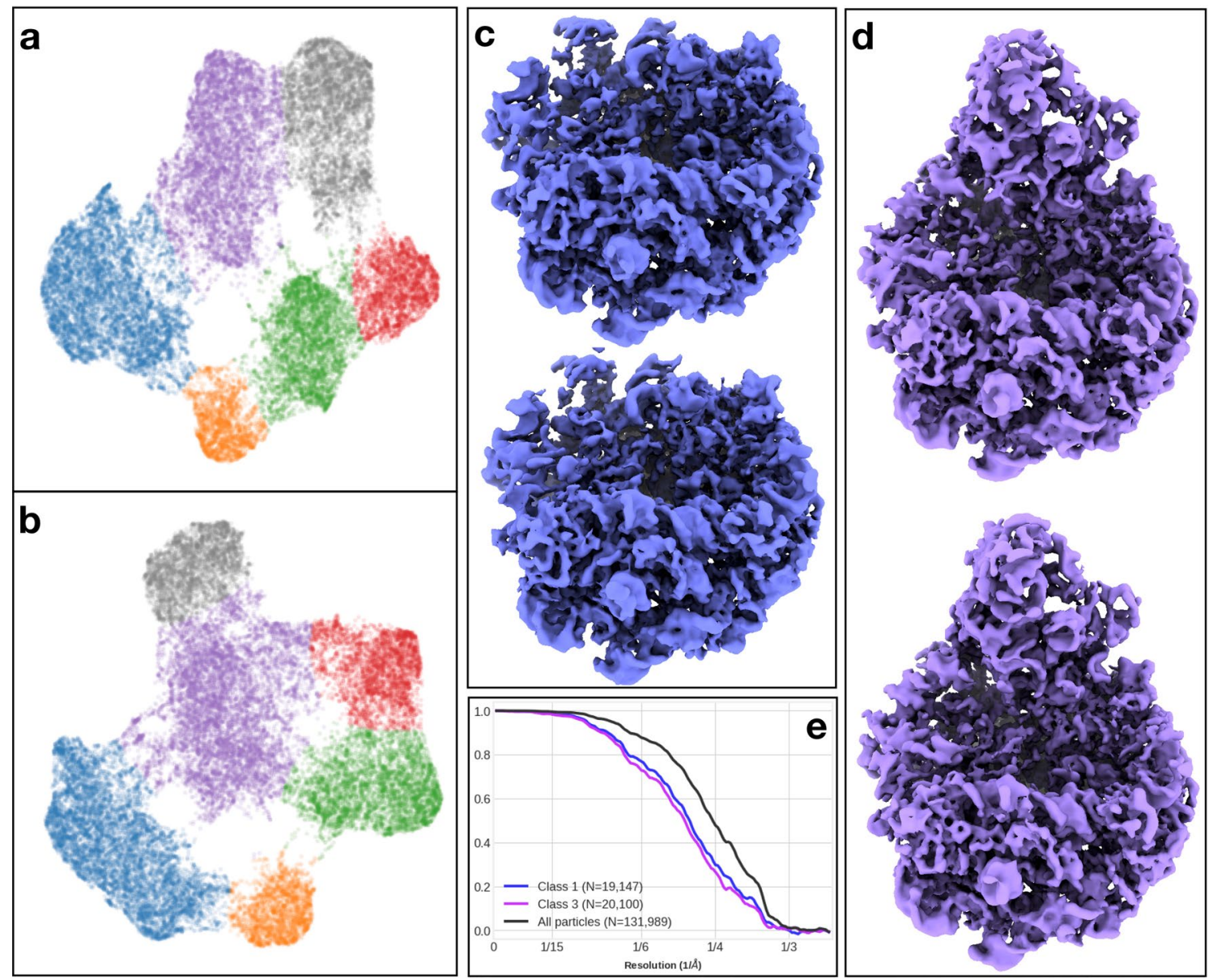

Extended Data Fig. 6 | Reproducibility of the method on the ribosome dataset. Reproducibility of the method on the ribosome dataset. $\mathbf{a}, \mathbf{b}, 2 \mathrm{D}$ conformation space embedding from heterogeneity analysis of two independent subsets of particles. The clusters are colored using the same scheme as Fig. 2. c, d, 3D class averages of particles in the same cluster from the two subsets. The maps are filtered by the local FSC between the two half maps. e, "Gold-standard" FSC curves of the full dataset (black), and the two classes shown in (c, $\mathbf{d}$,) (blue and purple). 


\section{NATURE METHODS}

ARTICLES
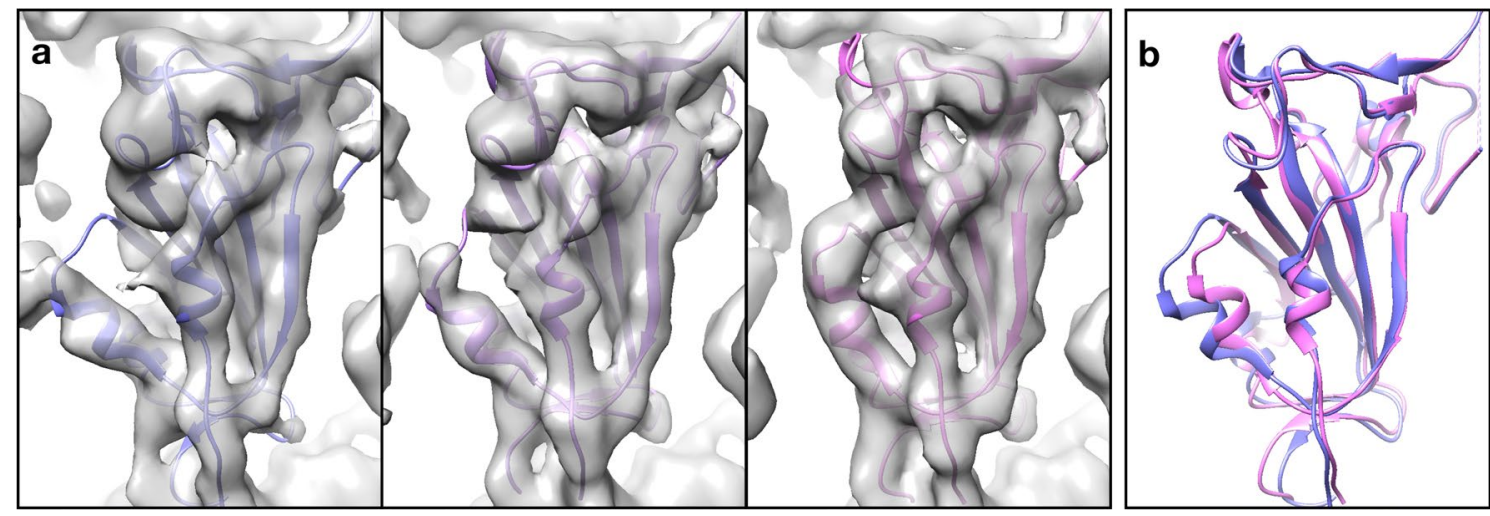

Extended Data Fig. 7 | Detailed structure of the default neural network. Detailed structure of the default neural network used for the examples shown in the paper. 


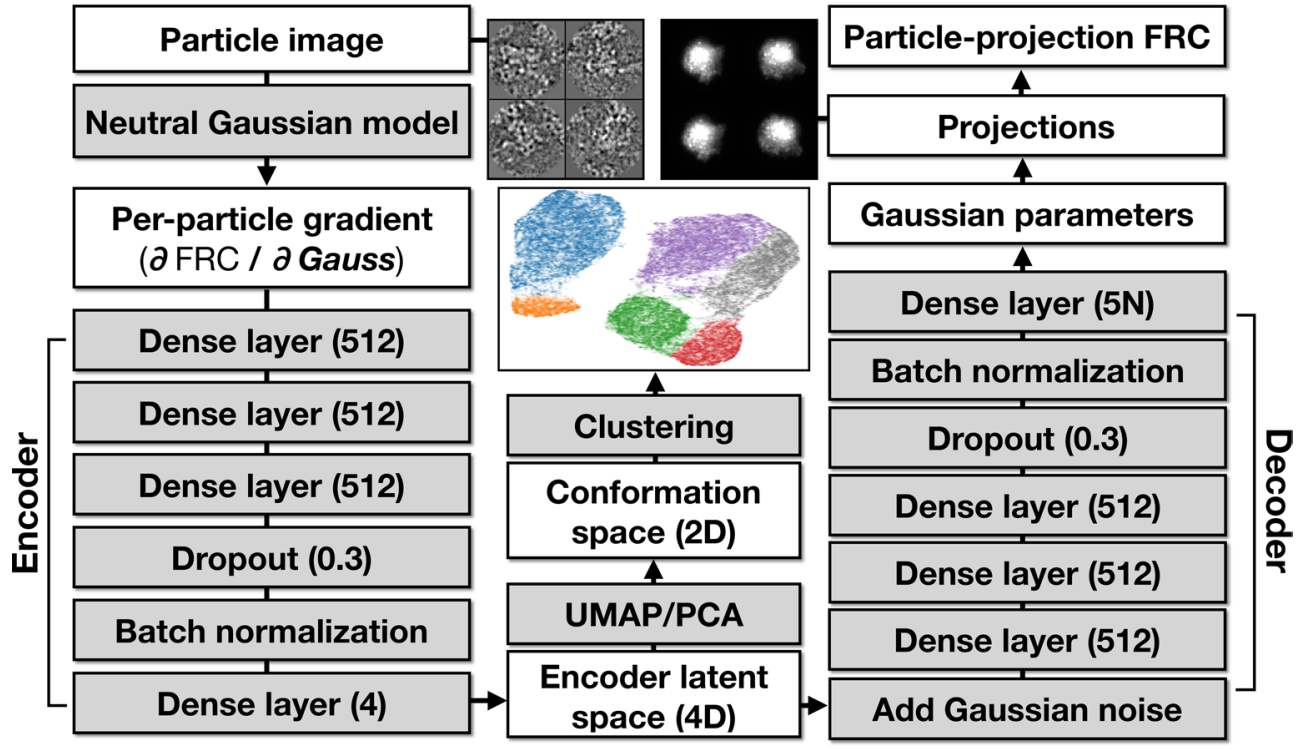

Extended Data Fig. 8 | Molecular models fit to the RBD of the SARS-COV-2 spike protein. Molecular models fit to individual 3D snapshots of the focused RBD of the SARS-COV-2 spike protein, along the trajectory of the first eigenvector (Fig. 5c-e). 


\section{Reporting Summary}

Nature Research wishes to improve the reproducibility of the work that we publish. This form provides structure for consistency and transparency in reporting. For further information on Nature Research policies, see our Editorial Policies and the Editorial Policy Checklist.

\section{Statistics}

For all statistical analyses, confirm that the following items are present in the figure legend, table legend, main text, or Methods section.

$\mathrm{n} / \mathrm{a}$ Confirmed

$\bigotimes$ The exact sample size $(n)$ for each experimental group/condition, given as a discrete number and unit of measurement

Х $\square$ A statement on whether measurements were taken from distinct samples or whether the same sample was measured repeatedly

Х The statistical test(s) used AND whether they are one- or two-sided

X Only common tests should be described solely by name; describe more complex techniques in the Methods section.

Х $\square$ A description of all covariates tested

Х $\square$ A description of any assumptions or corrections, such as tests of normality and adjustment for multiple comparisons

$\triangle$ A full description of the statistical parameters including central tendency (e.g. means) or other basic estimates (e.g. regression coefficient)

$X$ AND variation (e.g. standard deviation) or associated estimates of uncertainty (e.g. confidence intervals)

For null hypothesis testing, the test statistic (e.g. $F, t, r$ ) with confidence intervals, effect sizes, degrees of freedom and $P$ value noted

Give $P$ values as exact values whenever suitable.

Х $\square$ For Bayesian analysis, information on the choice of priors and Markov chain Monte Carlo settings

$\bigotimes \square$ For hierarchical and complex designs, identification of the appropriate level for tests and full reporting of outcomes

$\triangle \square$ Estimates of effect sizes (e.g. Cohen's $d$, Pearson's $r$ ), indicating how they were calculated

Our web collection on statistics for biologists contains articles on many of the points above.

\section{Software and code}

Policy information about availability of computer code

Data collection All data used is from the public EMPIAR data repository. No data collection is performed as a part of the paper.

Data analysis Data analysis was performed using EMAN2.91, FOSS software available from http://eman2.org with source code on GitHub (https:// github.com/cryoem/eman2).

For manuscripts utilizing custom algorithms or software that are central to the research but not yet described in published literature, software must be made available to editors and

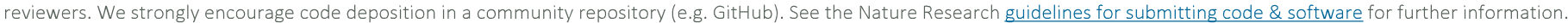

Data

Policy information about availability of data

All manuscripts must include a data availability statement. This statement should provide the following information, where applicable:

- Accession codes, unique identifiers, or web links for publicly available datasets

- A list of figures that have associated raw data

- A description of any restrictions on data availability

All data used is from the public EMPIAR data repository. The 50 S ribosome, spliceosome, and SARS-COV-2 datasets are available as EMPIAR-10076, EMPIAR-10180 and EMPIAR-10492 correspondingly. 
Please select the one below that is the best fit for your research. If you are not sure, read the appropriate sections before making your selection.

\ Life sciences

Behavioural \& social sciences

Ecological, evolutionary \& environmental sciences

For a reference copy of the document with all sections, see nature.com/documents/nr-reporting-summary-flat.pdf

\section{Life sciences study design}

All studies must disclose on these points even when the disclosure is negative.

Sample size The raw data is form the public EMPIAR data repository, and the sample size is determined by the authors depositing the dataset.

Data exclusions Clear non-protein particles, such as ice contamination, are removed before the analysis using the CNN-based particle picking tool in EMAN2.

Replication The result can be replicated using the same public dataset following the tutorial available through eman2.org. If all attempts at replication were successful. A reproducibility section is included in the Method part of the paper that describes the process in detail.

Randomization In the single particle refinement process, the dataset is evenly divided into two random subsets and aligned independently. The resolution is measured by the consistency of the results from the two subsets.

Blinding Blinding was not applicable to this study because this type of study does not use group allocation.

\section{Reporting for specific materials, systems and methods}

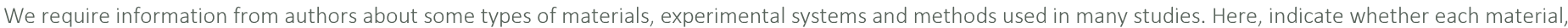

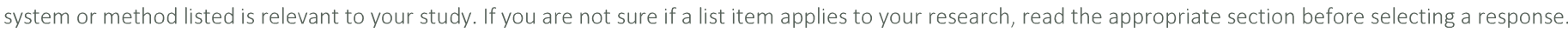

\begin{tabular}{|c|c|c|c|}
\hline \multicolumn{2}{|c|}{ Materials \& experimental systems } & \multicolumn{2}{|c|}{ Methods } \\
\hline $\mathrm{n} / \mathrm{a}$ & Involved in the study & $\mathrm{n} / \mathrm{a}$ & Involved in the study \\
\hline X & Antibodies & Х & ChIP-seq \\
\hline Х & Eukaryotic cell lines & Х & Flow cytometry \\
\hline Х & Palaeontology and archaeology & Х & MRI-based neuroimaging \\
\hline X & Animals and other organisms & & \\
\hline X & Human research participants & & \\
\hline Х & Clinical data & & \\
\hline Х & Dual use research of concern & & \\
\hline
\end{tabular}

